\title{
Blow-up criteria for Boussinesq system and MHD system and Landau-Lifshitz equations in a bounded domain
}

\author{
Jishan Fan' ${ }^{1}$ Wenjun Sun ${ }^{2}$ and Junping Yin ${ }^{2 *}$
}

\section{"Correspondence:}

yinjp829829@126.com

Institute of Applied Physics and

Computational Mathematics,

Beijing, 100088, P.R. China

Full list of author information is

available at the end of the article

\begin{abstract}
In this paper, we prove some blow-up criteria for the 3D Boussinesq system with zero heat conductivity and MHD system and Landau-Lifshitz equations in a bounded domain.
\end{abstract}

MSC: $35030 ; 76 D 03 ; 76 D 09$

Keywords: blow-up criterion; Boussinesq system; MHD system; Landau-Lifshitz equations

\section{Introduction}

Let $\Omega$ be a bounded, simply connected domain in $\mathbb{R}^{3}$ with smooth boundary $\partial \Omega$, and $v$ be the unit outward normal vector to $\partial \Omega$. First, we consider the regularity criterion of the Boussinesq system with zero heat conductivity:

$$
\begin{aligned}
& \operatorname{div} u=0, \\
& \partial_{t} u+u \cdot \nabla u+\nabla \pi-\Delta u=\theta e_{3}, \\
& \partial_{t} \theta+u \cdot \nabla \theta=0 \quad \text { in } \Omega \times(0, \infty), \\
& u \cdot v=0, \quad \operatorname{curl} u \times v=0 \quad \text { on } \partial \Omega \times(0, \infty), \\
& (u, \theta)(\cdot, 0)=\left(u_{0}, \theta_{0}\right) \quad \text { in } \Omega \subseteq \mathbb{R}^{3},
\end{aligned}
$$

where $u, \pi$, and $\theta$ denote the unknown velocity vector field, pressure scalar, and temperature scalar of the fluid, respectively. $\omega:=\operatorname{curl} u$ is the vorticity, and $e_{3}:=(0,0,1)^{t}$.

When $\theta=0,(1.1)$ and (1.2) are the well-known Navier-Stokes system. Giga [1], Kim [2], and Kang and Kim [3] have proved some Serrin-type regularity criteria.

The first aim of this paper is to prove a new regularity criterion for problem (1.1)-(1.5).

Theorem 1.1 Let $u_{0} \in H^{3}$ and $\theta_{0} \in W^{1, p}$ with $3<p \leq 6$ and $\operatorname{div} u_{0}=0$ in $\Omega$ and $u_{0} \cdot v=0$, $\operatorname{curl} u_{0} \times v=0$ on $\partial \Omega$. Let $(u, \theta)$ be a strong solution of problem (1.1)-(1.5). If $u$ satisfies

$$
\nabla u \in L^{1}(0, T ; \mathrm{BMO}(\Omega))
$$

(c) 2016 Fan et al. This article is distributed under the terms of the Creative Commons Attribution 4.0 International License (http://creativecommons.org/licenses/by/4.0/), which permits unrestricted use, distribution, and reproduction in any medium, provided you give appropriate credit to the original author(s) and the source, provide a link to the Creative Commons license, and indicate if changes were made. 
with $0<T<\infty$, then the solution $(u, \theta)$ can be extended beyond $T>0$. Here BMO denotes the space of bounded mean oscillation.

Secondly, we consider the blow-up criterion for the 3D MHD system

$$
\begin{aligned}
& \operatorname{div} u=\operatorname{div} b=0, \\
& \partial_{t} u+u \cdot \nabla u+\nabla\left(\pi+\frac{1}{2}|b|^{2}\right)-\Delta u=b \cdot \nabla b, \\
& \partial_{t} b+u \cdot \nabla b-b \cdot \nabla u=\Delta b \quad \text { in } \Omega \times(0, \infty), \\
& u \cdot v=0, \quad \operatorname{curl} u \times v=0, \quad b \cdot v=0, \quad \operatorname{curl} b \times v=0 \\
& \quad \text { on } \partial \Omega \times(0, \infty), \\
& (u, b)(\cdot, 0)=\left(u_{0}, b_{0}\right) \quad \text { in } \Omega \subseteq \mathbb{R}^{3} .
\end{aligned}
$$

Here $b$ is the magnetic field of the fluid.

It is well known that problem (1.7)-(1.11) has a unique local strong solution [4]. But whether this local solution can exist globally is an outstanding problem. Kang and Kim [3] proved some Serrin-type regularity criteria.

The second aim of this paper is to prove a new regularity criterion for problem (1.7)(1.11).

Theorem 1.2 Let $u_{0}, b_{0} \in H^{3}$ with $\operatorname{div} u_{0}=\operatorname{div} b_{0}=0$ in $\Omega$ and $u_{0} \cdot v=b_{0} \cdot v=0$, curl $u_{0} \times$ $v=\operatorname{curl} b_{0} \times v=0$ on $\partial \Omega$. Let $(u, b)$ be a strong solution to problem (1.7)-(1.11).If (1.6) holds, then the solution $(u, b)$ can be extended beyond $T>0$.

Remark 1.1 When $\Omega:=\mathbb{R}^{3}$, our result gives the well-known regularity criterion

$$
\omega:=\operatorname{curl} u \in L^{1}\left(0, T ; \dot{B}_{\infty, \infty}^{0}\right)
$$

but the method of proof we use is different from that in $[5,6]$. Here $\dot{B}_{\infty, \infty}^{0}$ denotes the homogeneous Besov space [7].

Next, we consider the following 3D density-dependent MHD equations:

$$
\begin{aligned}
& \operatorname{div} u=\operatorname{div} b=0, \\
& \partial_{t} \rho+\operatorname{div}(\rho u)=0, \\
& \partial_{t}(\rho u)+\operatorname{div}(\rho u \otimes u)+\nabla\left(\pi+\frac{1}{2}|b|^{2}\right)-\Delta u=b \cdot \nabla b, \\
& \partial_{t} b+u \cdot \nabla b-b \cdot \nabla u=\Delta b \quad \operatorname{in} \Omega \times(0, \infty), \\
& u=0, \quad b \cdot v=0, \quad \operatorname{curl} b \times v=0 \quad \text { on } \partial \Omega \times(0, \infty), \\
& (\rho, \rho u, b)(\cdot, 0)=\left(\rho_{0}, \rho_{0} u_{0}, b_{0}\right) \quad \text { in } \Omega \subset \mathbb{R}^{3} .
\end{aligned}
$$


For this problem, $\mathrm{Wu}[8]$ proved that if the initial data $\rho_{0}, u_{0}$, and $b_{0}$ satisfy

$$
\begin{aligned}
& 0 \leq \rho_{0} \in H^{2}, \quad u_{0} \in H_{0}^{1} \cap H^{2}, \quad b_{0} \in H^{2}, \\
& -\Delta u_{0}+\nabla\left(\pi_{0}+\frac{1}{2}\left|b_{0}\right|^{2}\right)=b_{0} \cdot \nabla b_{0}+\sqrt{\rho_{0}} g
\end{aligned}
$$

for some $\left(\pi_{0}, g\right) \in H^{1} \times L^{2}$, then there exists a positive time $T_{*}$ and a unique strong solution $(\rho, u, b)$ to problem (1.12)-(1.17) such that

$$
\begin{aligned}
& \rho \in C\left(\left[0, T_{*}\right] ; H^{2}\right), \quad u \in C\left(\left[0, T_{*}\right] ; H_{0}^{1} \cap H^{2}\right) \cap L^{2}\left(0, T_{*} ; H^{2}\right), \\
& u_{t} \in L^{2}\left(0, T_{*} ; H_{0}^{1}\right), \quad \sqrt{\rho} u_{t} \in L^{\infty}\left(0, T_{*} ; L^{2}\right), \\
& b \in L^{\infty}\left(0, T_{*} ; H^{2}\right) \cap L^{2}\left(0, T_{*} ; H^{3}\right), \quad b_{t} \in L^{\infty}\left(0, T_{*} ; L^{2}\right) \cap L^{2}\left(0, T_{*} ; H^{1}\right) .
\end{aligned}
$$

When $b=0$, Kim [2] proved the following regularity criterion:

$$
u \in L^{\frac{2 s}{s-3}}\left(0, T ; L_{w}^{s}(\Omega)\right) \quad \text { with } 3<s \leq \infty .
$$

Here $L_{w}^{s}$ denotes the weak- $L^{s}$ space, and $L_{w}^{\infty}=L^{\infty}$.

The aim of this paper is to refine (1.20) as follows.

Theorem 1.3 Let $\rho_{0}, u_{0}$, and $b_{0}$ satisfy $(1.18)$. Let $(\rho, u, b)$ be a strong solution of problem (1.12)-(1.17) in the class (1.19). Suppose that u satisfies one of the following two conditions:

$$
\begin{aligned}
& \text { (i) } \int_{0}^{T} \frac{\|u(t)\|_{L_{w}^{s}}^{\frac{2 s}{s-3}}}{1+\log \left(e+\|u(t)\|_{L_{w}^{s}}\right)} d t<\infty \quad \text { with } 3<s \leq \infty, \\
& \text { (ii) } u \in L^{2}(0, T ; \operatorname{BMO}(\Omega))
\end{aligned}
$$

with $0<T<\infty$. Then the solution $(\rho, u, b)$ can be extended beyond $T>0$.

Finally, we consider the 3D Landau-Lifshitz system:

$$
\begin{aligned}
& \partial_{t} d-\Delta d=d|\nabla d|^{2}+d \times \Delta d, \quad|d|=1 \quad \text { in } \Omega \times(0, \infty), \\
& \partial_{\nu} d=0 \quad \text { on } \partial \Omega \times(0, \infty), \\
& d(\cdot, 0)=d_{0}, \quad\left|d_{0}\right|=1 \quad \text { in } \Omega \subseteq \mathbb{R}^{3} .
\end{aligned}
$$

Carbou and Fabrie [9] showed the existence and uniqueness of local smooth solutions. When $\Omega:=\mathbb{R}^{n}(n=2,3,4)$, Fan and Ozawa [10] proved some regularity criteria. The aim of this paper is to prove a logarithmic blow-up criterion for problem (1.23)-(1.25) when $\Omega$ is a bounded domain. We will prove the following:

Theorem 1.4 Let $d_{0} \in H^{3}(\Omega)$ with $\left|d_{0}\right|=1$ in $\Omega$ and $\partial_{\nu} d_{0}=0$ on $\partial \Omega$. Let $d$ be a local smooth solution to problem (1.23)-(1.25). If d satisfies

$$
\int_{0}^{T} \frac{\|\nabla d\|_{L^{q}}^{\frac{2 q}{q-3}}}{1+\log \left(e+\|\nabla d\|_{L^{q}}\right)} d t<\infty \quad \text { with } 3<q \leq \infty
$$

and $0<T<\infty$, then the solution can be extended beyond $T>0$. 
In Section 2, we give some preliminary lemmas, which will be used in the following sections. The proof of Theorem 1.1 for problem (1.1)-(1.5) will be given in Section 3. The new regularly criterion of Theorem 1.2 for the 3D MHD problem (1.7)-(1.11) will be proved in Section 4. In Section 5, we prove Theorem 1.3, and in Section 6, we give the main proof of final Theorem 1.4.

\section{Preliminary lemmas}

In the following proofs, we will use the logarithmic Sobolev inequality [11]

$$
\|\nabla u\|_{L^{\infty}} \leq C\left(1+\|\nabla u\|_{\mathrm{BMO}} \log \left(e+\|u\|_{W^{s, p}}\right)\right) \quad \text { with } s>1+\frac{3}{p}
$$

and the following three lemmas.

Lemma 2.1 ([12]) Let $\Omega \subseteq \mathbb{R}^{3}$ be a smooth bounded domain, let $b: \Omega \rightarrow \mathbb{R}^{3}$ be a smooth vector field, and let $1<p<\infty$. Then

$$
\begin{aligned}
-\int_{\Omega} \Delta b \cdot b|b|^{p-2} d x= & \frac{1}{2} \int_{\Omega}|b|^{p-2}|\nabla b|^{2} d x+\left.\left.4 \frac{p-2}{p^{2}} \int_{\Omega}|\nabla| b\right|^{\frac{p}{2}}\right|^{2} d x \\
& -\int_{\partial \Omega}|b|^{p-2}(b \cdot \nabla) b \cdot v d \sigma-\int_{\partial \Omega}|b|^{p-2}(\operatorname{curl} b \times v) \cdot b d \sigma .
\end{aligned}
$$

Lemma $2.2([13,14])$ Let $\Omega$ be a smooth and bounded open set, and let $1<p<\infty$. Then we have the estimate

$$
\|b\|_{L^{p}(\partial \Omega)} \leq C\|b\|_{L^{p}(\Omega)}^{1-\frac{1}{p}}\|b\|_{W^{1, p}(\Omega)}^{\frac{1}{p}}
$$

for all $b \in W^{1, p}(\Omega)$.

Lemma 2.3 We have

$$
\|f\|_{L^{\infty}(\Omega)} \leq C\left(1+\|f\|_{\mathrm{BMO}(\Omega)} \log ^{\frac{1}{2}}\left(e+\|f\|_{W^{1,4}(\Omega)}\right)\right)
$$

for all $f \in W_{0}^{1,4}(\Omega)$.

Proof When $\Omega:=\mathbb{R}^{3},(2.4)$ is proved by Ogawa [15]. For a bounded domain $\Omega$ in $\mathbb{R}^{3}$, we define

$$
\tilde{f}:= \begin{cases}f & \text { in } \Omega, \\ 0 & \text { in } \Omega^{c}:=\mathbb{R}^{3} \backslash \Omega .\end{cases}
$$

Then we have [13], p.71,

$$
\|\tilde{f}\|_{W^{1,4}\left(\mathbb{R}^{3}\right)}=\|f\|_{W^{1,4}(\Omega)},
$$

and it is obvious that

$$
\|\tilde{f}\|_{L^{\infty}\left(\mathbb{R}^{3}\right)}=\|f\|_{L^{\infty}(\Omega)},\|\tilde{f}\|_{\mathrm{BMO}\left(\mathbb{R}^{3}\right)}=\|f\|_{\mathrm{BMO}(\Omega)}
$$

Thus, (2.4) is proved. 
Finally, when $b$ satisfies $b \cdot v=0$ on $\partial \Omega$, we will also use the identity

$$
(b \cdot \nabla) b \cdot v=-(b \cdot \nabla) v \cdot b \quad \text { on } \partial \Omega
$$

for any sufficiently smooth vector field $b$.

\section{Proof of Theorem 1.1}

Since it is easy to prove that problem (1.1)-(1.5) has a unique local-in-time strong solution, we omit the details. We only need to establish a priori estimates.

First, thanks to the maximum principle, it follows from (1.1) and (1.3) that

$$
\|\theta\|_{L^{\infty}\left(0, T ; L^{\infty}\right)} \leq C
$$

Testing (1.2) by $u$ and using (1.1) and (3.1), we see that

$$
\frac{1}{2} \frac{d}{d t} \int_{\Omega} u^{2} d x+\int_{\Omega}|\operatorname{curl} u|^{2} d x \leq \int_{\Omega} \theta e_{3} \cdot u d x \leq \frac{1}{2} \int_{\Omega} \theta^{2} d x+\frac{1}{2} \int_{\Omega} u^{2} d x,
$$

which gives

$$
\|u\|_{L^{\infty}\left(0, T ; L^{2}\right)}+\|u\|_{L^{2}\left(0, T ; H^{1}\right)} \leq C .
$$

Applying curl to (1.2) and setting $\omega:=\operatorname{curl} u$, we find that

$$
\partial_{t} \omega+u \cdot \nabla \omega-\Delta \omega=\omega \cdot \nabla u+\operatorname{curl}\left(\theta e_{3}\right)
$$

Testing (3.3) by $\omega$ and using (1.1) and (3.1), we infer that

$$
\begin{aligned}
\frac{1}{2} \frac{d}{d t} \int_{\Omega}|\omega|^{2} d x+\int_{\Omega}|\operatorname{curl} \omega|^{2} d x & =\int_{\Omega}(\omega \cdot \nabla) u \cdot \omega d x+\int_{\Omega} \theta e_{3} \operatorname{curl} \omega d x \\
& \leq\|\nabla u\|_{L^{\infty}} \int_{\Omega} \omega^{2} d x+\frac{1}{2} \int_{\Omega}|\operatorname{curl} \omega|^{2} d x+C
\end{aligned}
$$

which implies

$$
\begin{aligned}
\frac{d}{d t} \int_{\Omega}|\omega|^{2} d x+\int_{\Omega}|\operatorname{curl} \omega|^{2} d x & \leq C\|\nabla u\|_{L^{\infty}} \int_{\Omega}|\omega|^{2} d x+C \\
& \leq C\left(1+\|\nabla u\|_{\text {Вмо }}\right) \log \left(e+\|u\|_{H^{3}}\right) \int_{\Omega}|\omega|^{2} d x+C,
\end{aligned}
$$

and therefore

$$
\int_{\Omega}|\omega|^{2} d x+\int_{t_{0}}^{t}\|\operatorname{curl} \omega\|_{L^{2}}^{2} d \tau \leq C(e+y)^{C_{0} \epsilon}
$$

provided that

$$
\int_{t_{0}}^{t}\|\nabla u\|_{\text {ВмO }} d \tau \leq \epsilon \ll 1
$$

and $y(t):=\sup _{\left[t_{0}, t\right]}\|u\|_{H^{3}}$ for any $0<t_{0} \leq t \leq T$, and $C_{0}$ is an absolute constant. 
Applying $\partial_{t}$ to (1.2), we deduce that

$$
\partial_{t}^{2} u+u \cdot \nabla u_{t}+\nabla \pi_{t}-\Delta u_{t}=-u_{t} \cdot \nabla u+\theta_{t} e_{3}
$$

Testing (3.6) by $u_{t}$ and using (1.1), (1.3), (3.1), and (3.2), we derive

$$
\begin{aligned}
\frac{1}{2} & \frac{d}{d t} \int_{\Omega}\left|u_{t}\right|^{2} d x+\int_{\Omega}\left|\operatorname{curl} u_{t}\right|^{2} d x \\
& =-\int_{\Omega} u_{t} \cdot \nabla u \cdot u_{t} d x+\int_{\Omega} \theta_{t} e_{3} u_{t} d x \\
& =-\int_{\Omega} u_{t} \cdot \nabla u \cdot u_{t} d x-\int_{\Omega} \operatorname{div}(u \theta) e_{3} u_{t} d x \\
& =-\int_{\Omega} u_{t} \cdot \nabla u \cdot u_{t} d x+\int_{\Omega} u \theta \nabla\left(e_{3} u_{t}\right) d x \\
& \leq\|\nabla u\|_{L^{\infty}} \int_{\Omega}\left|u_{t}\right|^{2} d x+\frac{1}{2} \int_{\Omega}\left|\operatorname{curl} u_{t}\right|^{2} d x+C \\
& \leq C\left(1+\|\nabla u\|_{\mathrm{BMO}}\right) \log (e+y) \int_{\Omega}\left|u_{t}\right|^{2} d x+\frac{1}{2} \int_{\Omega}\left|\operatorname{curl} u_{t}\right|^{2} d x+C,
\end{aligned}
$$

which yields

$$
\int_{\Omega}\left|u_{t}\right|^{2} d x+\int_{t_{0}}^{t} \int_{\Omega}\left|\operatorname{curl} u_{t}\right|^{2} d x d \tau \leq C(e+y)^{C_{0} \epsilon}
$$

On the other hand, thanks to the $H^{2}$-theory of the Stokes system, if follows from (1.2), (3.1), (3.4), and (3.7) that

$$
\begin{aligned}
\|u\|_{H^{2}} & \leq C\|-\Delta u+\nabla \pi\|_{L^{2}} \\
& \leq C\left\|\partial_{t} u+u \cdot \nabla u-\theta e_{3}\right\|_{L^{2}} \\
& \leq C\left\|u_{t}\right\|_{L^{2}}+C\|u\|_{L^{6}}\|\nabla u\|_{L^{3}}+C\|\theta\|_{L^{2}} \\
& \leq C\left\|u_{t}\right\|_{L^{2}}+C\|\nabla u\|_{L^{2}}^{\frac{3}{2}}\|u\|_{H^{2}}^{\frac{1}{2}}+C,
\end{aligned}
$$

which implies

$$
\|u\|_{H^{2}} \leq C\left\|u_{t}\right\|_{L^{2}}+C\|\nabla u\|_{L^{2}}^{3}+C \leq C(e+y)^{C_{0} \epsilon} .
$$

Applying $\nabla$ to (1.3), testing by $|\nabla \theta|^{p-2} \nabla \theta(2 \leq p<\infty)$, and using (1.1), we get

$$
\begin{aligned}
\frac{d}{d t}\|\nabla \theta\|_{L^{p}} & \leq C\|\nabla u\|_{L^{\infty}}\|\nabla \theta\|_{L^{p}} \\
& \leq C\left(1+\|\nabla u\|_{\mathrm{BMO}}\right) \log (e+y)\|\nabla \theta\|_{L^{p}}
\end{aligned}
$$

which leads to

$$
\|\nabla \theta\|_{L^{\infty}\left(t_{0}, t ; L^{p}\right)} \leq C(e+y)^{C_{0} \epsilon} \quad \text { with } 2 \leq p<\infty .
$$


Testing (3.6) by $-\Delta u_{t}+\nabla \pi_{t}$ and using (1.1), (1.3), (3.7), (3.8), and (3.9), we obtain

$$
\begin{aligned}
& \frac{1}{2} \frac{d}{d t} \int_{\Omega}\left|\operatorname{curl} u_{t}\right|^{2} d x+\int_{\Omega}\left|-\Delta u_{t}+\nabla \pi_{t}\right|^{2} d x \\
& \quad=\int_{\Omega}\left(-u_{t} \cdot \nabla u+\theta_{t} e_{3}-u \cdot \nabla u_{t}\right)\left(-\Delta u_{t}+\nabla \pi_{t}\right) d x \\
& \quad \leq\left(\|\nabla u\|_{L^{6}}\left\|u_{t}\right\|_{L^{3}}+\|u\|_{L^{\infty}}\|\nabla \theta\|_{L^{2}}+\|u\|_{L^{\infty}}\left\|\nabla u_{t}\right\|_{L^{2}}\right)\left\|-\Delta u_{t}+\nabla \pi_{t}\right\|_{L^{2}} \\
& \quad \leq\|u\|_{H^{2}}\left(\left\|u_{t}\right\|_{H^{1}}+\|\nabla \theta\|_{L^{2}}\right)\left\|-\Delta u_{t}+\nabla \pi_{t}\right\|_{L^{2}} \\
& \quad \leq \frac{1}{2}\left\|-\Delta u_{t}+\nabla \pi_{t}\right\|_{L^{2}}^{2}+C\|u\|_{H^{2}}^{2}\left(\left\|u_{t}\right\|_{H^{1}}^{2}+\|\nabla \theta\|_{L^{2}}^{2}\right),
\end{aligned}
$$

which leads to

$$
\int_{\Omega}\left|\operatorname{curl} u_{t}\right|^{2} d x+\int_{t_{0}}^{t}\left\|u_{t}\right\|_{H^{2}}^{2} d \tau \leq C(e+y)^{C_{0} \epsilon}
$$

On the other hand, if follows from (3.3), (3.10), (3.9), and (3.8) that

$$
\begin{aligned}
\|u\|_{H^{3}} & \leq C\left(1+\|\Delta \omega\|_{L^{2}}\right) \\
& \leq C\left(1+\left\|\partial_{t} \omega+u \cdot \nabla \omega-\omega \cdot \nabla u-\operatorname{curl}\left(\theta e_{3}\right)\right\|_{L^{2}}\right) \\
& \leq C\left(1+\left\|\partial_{t} \omega\right\|_{L^{2}}+\|u\|_{L^{\infty}}\|\nabla \omega\|_{L^{2}}+\|\omega\|_{L^{4}}\|\nabla u\|_{L^{4}}+\|\nabla \theta\|_{L^{2}}\right) \\
& \leq C(e+y)^{C_{0} \epsilon}
\end{aligned}
$$

which gives

$$
\|u\|_{L^{\infty}\left(0, T ; H^{3}\right)} \leq C
$$

and

$$
\|\theta\|_{L^{\infty}\left(0, T ; W^{1, p}\right)} \leq C \quad \text { with } 3 \leq p \leq 6
$$

This completes the proof of Theorem 1.1.

\section{Proof of Theorem 1.2}

We only need to prove a priori estimates.

First, testing (1.8) by $u$ and using (1.7), we see that

$$
\frac{1}{2} \frac{d}{d t} \int_{\Omega} u^{2} d x+\int_{\Omega}|\operatorname{curl} u|^{2} d x=\int_{\Omega}(b \cdot \nabla) b \cdot u d x .
$$

Testing (1.9) by $b$ and using (1.7), we find that

$$
\frac{1}{2} \frac{d}{d t} \int_{\Omega} b^{2} d x+\int_{\Omega}|\operatorname{curl} b|^{2} d x=\int_{\Omega}(b \cdot \nabla) u \cdot b d x
$$

Summing up (4.1) and (4.2), we get the well-known energy inequality

$$
\frac{1}{2} \frac{d}{d t} \int_{\Omega}\left(u^{2}+b^{2}\right) d x+\int_{\Omega}\left(|\operatorname{curl} u|^{2}+|\operatorname{curl} b|^{2}\right) d x \leq 0
$$


Testing (1.9) by $|b|^{p-2} b(2 \leq p \leq 6)$ and using (1.7), (2.2), (2.3), and (2.5), we derive

$$
\begin{aligned}
\frac{1}{p} & \frac{d}{d t} \int_{\Omega}|b|^{p} d x+\frac{1}{2} \int_{\Omega}|b|^{p-2}|\nabla b|^{2} d x+\left.\left.4 \frac{p-2}{p^{2}} \int_{\Omega}|\nabla| b\right|^{\frac{p}{2}}\right|^{2} d x \\
& =-\int_{\partial \Omega}|b|^{p-2}(b \cdot \nabla) v \cdot b d \sigma+\int_{\Omega} b \cdot \nabla u \cdot|b|^{p-2} b d x \\
& \leq C \int_{\partial \Omega}|b|^{p} d x+\|\nabla u\|_{L^{\infty}} \int_{\Omega}|b|^{p} d x \\
& \leq\left.\left. 2 \frac{p-2}{p^{2}} \int_{\Omega}|\nabla| b\right|^{\frac{p}{2}}\right|^{2} d x+C\left(1+\|\nabla u\|_{L^{\infty}}\right) \int_{\Omega}|b|^{p} d x \\
& \leq\left.\left. 2 \frac{p-2}{p^{2}} \int_{\Omega}|\nabla| b\right|^{\frac{p}{2}}\right|^{2} d x+C\left(1+\|\nabla u\|_{\mathrm{BMO}}\right) \int_{\Omega}|b|^{p} d x \log (e+y)
\end{aligned}
$$

which implies

$$
\|b\|_{L^{\infty}\left(t_{0}, t ; L^{p}\right)}+\int_{t_{0}}^{t} \int_{\Omega}|b|^{2}|\nabla b|^{2} d x d \tau \leq C(e+y)^{C_{0} \epsilon} \quad \text { with } 2 \leq p \leq 6
$$

with the same $y$ and $\epsilon$ as in (3.5).

Taking curl to (1.8) and (1.9), respectively, and setting $\omega:=\operatorname{curl} u$ and $j:=\operatorname{curl} b$, we infer that

$$
\begin{aligned}
& \partial_{t} \omega+u \cdot \nabla \omega-\Delta \omega=\omega \cdot \nabla u+b \cdot \nabla j+\sum_{i} \nabla b_{i} \times \partial_{i} b \\
& \partial_{t} j+u \cdot \nabla j-\Delta j=b \cdot \nabla \omega+\sum_{i} \nabla b_{i} \times \partial_{i} u-\sum_{i} \nabla u_{i} \times \partial_{i} b .
\end{aligned}
$$

Testing (4.5) and (4.6) by $\omega$ and $j$, respectively, summing up the result, and using (1.7), we have

$$
\begin{aligned}
& \frac{1}{2} \frac{d}{d t} \int_{\Omega}\left(\omega^{2}+j^{2}\right) d x+\int_{\Omega}\left(|\operatorname{curl} \omega|^{2}+|\operatorname{curl} j|^{2}\right) d x \\
& =\int_{\Omega}(\omega \cdot \nabla) u \cdot \omega d x+\sum_{i} \int_{\Omega}\left(\nabla b_{i} \times \partial_{i} b\right) \omega d x \\
& \quad+\sum_{i} \int_{\Omega}\left(\nabla b_{i} \times \partial_{i} u\right) \cdot j d x-\sum_{i} \int_{\Omega}\left(\nabla u_{i} \times \partial_{i} b\right) \cdot j d x \\
& \leq C\|\nabla u\|_{L^{\infty}} \int_{\Omega}\left(\omega^{2}+j^{2}\right) d x \\
& \leq C\left(1+\|\nabla u\|_{\mathrm{BMO}}\right) \int_{\Omega}\left(\omega^{2}+j^{2}\right) d x \log (e+y)
\end{aligned}
$$

which implies

$$
\int_{\Omega}\left(\omega^{2}+j^{2}\right) d x+\int_{t_{0}}^{t} \int_{\Omega}\left(|\operatorname{curl} \omega|^{2}+|\operatorname{curl} j|^{2}\right) d x d \tau \leq C(e+y)^{C_{0} \epsilon} .
$$


Thus, it follows from (1.8), (1.9), and (4.7) that

$$
\int_{t_{0}}^{t} \int_{\Omega}\left(\left|u_{t}\right|^{2}+\left|b_{t}\right|^{2}\right) d x d \tau \leq C(e+y)^{C_{0} \epsilon}
$$

Applying $\partial_{t}$ to (1.8), we have

$$
\partial_{t}^{2} u+u \cdot \nabla u_{t}+\nabla \pi_{t}-\Delta u_{t}=\operatorname{div}(b \otimes b)_{t}-u_{t} \cdot \nabla u
$$

Testing (4.9) by $u_{t}$ and using (1.7), we get

$$
\begin{aligned}
& \frac{1}{2} \frac{d}{d t} \int_{\Omega}\left|u_{t}\right|^{2} d x+\int_{\Omega}\left|\operatorname{curl} u_{t}\right|^{2} d x \\
& \quad=-\sum_{i, j} \int_{\Omega}\left(b^{i} b^{j}\right)_{t} \partial_{j} u_{t}^{i} d x-\int_{\Omega} u_{t} \cdot \nabla u \cdot u_{t} d x \\
& \quad \leq C\left\|b_{t}\right\|_{L^{3}}\|b\|_{L^{6}}\left\|\nabla u_{t}\right\|_{L^{2}}+\|\nabla u\|_{L^{2}}\left\|u_{t}\right\|_{L^{4}}^{2} \\
& \quad \leq C\left\|b_{t}\right\|_{L^{2}}^{\frac{1}{2}}\left\|\operatorname{curl} b_{t}\right\|_{L^{2}}^{\frac{1}{2}}\left\|\operatorname{curl} u_{t}\right\|_{L^{2}}\|b\|_{L^{6}}+C\|\nabla u\|_{L^{2}}\left\|u_{t}\right\|_{L^{2}}^{\frac{1}{2}}\left\|\operatorname{curl} u_{t}\right\|_{L^{2}}^{\frac{3}{2}} \\
& \quad \leq \delta\left\|\operatorname{curl} u_{t}\right\|_{L^{2}}^{2}+\delta\left\|\operatorname{curl} b_{t}\right\|_{L^{2}}^{2}+C\left\|b_{t}\right\|_{L^{2}}^{2}\|b\|_{L^{6}}^{4}+C\|\nabla u\|_{L^{2}}^{4}\left\|u_{t}\right\|_{L^{2}}^{2}
\end{aligned}
$$

for any $\delta \in(0,1)$.

Applying $\partial_{t}$ to (1.9), we have

$$
\partial_{t}^{2} b+u \cdot \nabla b_{t}-\Delta b_{t}=b_{t} \cdot \nabla u+b \cdot \nabla u_{t}-u_{t} \cdot \nabla b
$$

Testing (4.11) by $b_{t}$ and using (1.7), we deduce that

$$
\begin{aligned}
& \frac{1}{2} \frac{d}{d t} \int_{\Omega}\left|b_{t}\right|^{2} d x+\int_{\Omega}\left|\operatorname{curl} b_{t}\right|^{2} d x \\
& =\int_{\Omega}\left(b_{t} \cdot \nabla u+b \cdot \nabla u_{t}-u_{t} \cdot \nabla b\right) b_{t} d x \\
& \leq\|\nabla u\|_{L^{2}}\left\|b_{t}\right\|_{L^{4}}^{2}+\|b\|_{L^{6}}\left\|\nabla u_{t}\right\|_{L^{2}}\left\|b_{t}\right\|_{L^{3}}+\|\nabla b\|_{L^{2}}\left\|u_{t}\right\|_{L^{4}}\left\|b_{t}\right\|_{L^{4}} \\
& \leq \delta\left\|\operatorname{curl} b_{t}\right\|_{L^{2}}^{2}+\delta\left\|\operatorname{curl} u_{t}\right\|_{L^{2}}^{2} \\
& \quad+C\|\nabla u\|_{L^{2}}^{4}\left\|b_{t}\right\|_{L^{2}}^{2}+C\|b\|_{L^{6}}^{4}\left\|b_{t}\right\|_{L^{2}}^{2}+C\|\nabla b\|_{L^{2}}^{4}\left(\left\|u_{t}\right\|_{L^{2}}^{2}+\left\|b_{t}\right\|_{L^{2}}^{2}\right)
\end{aligned}
$$

for any $\delta \in(0,1)$.

Combining (4.10) and (4.12), taking $\delta$ small enough, and using (4.7) and (4.8), we have

$$
\int_{\Omega}\left(\left|u_{t}\right|^{2}+\left|b_{t}\right|^{2}\right) d x+\int_{t_{0}}^{t} \int_{\Omega}\left(\left|\operatorname{curl} u_{t}\right|^{2}+\left|\operatorname{curl} b_{t}\right|^{2}\right) d x d \tau \leq C(e+y)^{C_{0} \epsilon}
$$

It follows from (1.8), (1.9), (4.7), and (4.13) that

$$
\|u\|_{L^{\infty}\left(t_{0}, t ; H^{2}\right)}+\|b\|_{L^{\infty}\left(t_{0}, t ; H^{2}\right)} \leq C(e+y)^{C_{0} \epsilon} .
$$


Testing (4.9) by $\nabla\left(\pi+\frac{1}{2}|b|^{2}\right)_{t}-\Delta u_{t}$ and using (1.7), we find that

$$
\begin{aligned}
\frac{1}{2} & \frac{d}{d t} \int_{\Omega}\left|\operatorname{curl} u_{t}\right|^{2} d x+\int_{\Omega}\left|\nabla\left(\pi+\frac{1}{2}|b|^{2}\right)_{t}-\Delta u_{t}\right|^{2} d x \\
= & \int_{\Omega}\left((b \cdot \nabla b)_{t}-u_{t} \cdot \nabla u-u \cdot \nabla u_{t}\right)\left(\nabla\left(\pi+\frac{1}{2}|b|^{2}\right)_{t}-\Delta u_{t}\right) d x \\
\leq & C\left(\|b\|_{L^{\infty}}\left\|\nabla b_{t}\right\|_{L^{2}}+\left\|b_{t}\right\|_{L^{6}}\|\nabla b\|_{L^{3}}+\left\|u_{t}\right\|_{L^{6}}\|\nabla u\|_{L^{3}}\right. \\
& \left.+\|u\|_{L^{\infty}}\left\|\nabla u_{t}\right\|_{L^{2}}\right)\left\|\nabla\left(\pi+\frac{1}{2}|b|^{2}\right)_{t}-\Delta u_{t}\right\|_{L^{2}} \\
\leq & \frac{1}{4}\left\|\nabla\left(\pi+\frac{1}{2}|b|^{2}\right)_{t}-\Delta u_{t}\right\|_{L^{2}}^{2}+C\left(\|u\|_{L^{\infty}}^{2}+\|\nabla u\|_{L^{3}}^{2}\right)\left\|\nabla u_{t}\right\|_{L^{2}}^{2} \\
& +C\left(\|b\|_{L^{\infty}}^{2}+\|\nabla b\|_{L^{3}}^{2}\right)\left\|\nabla b_{t}\right\|_{L^{2}}^{2} .
\end{aligned}
$$

Similarly, testing (4.11) by $-\Delta b_{t}$, we infer that

$$
\begin{aligned}
& \frac{1}{2} \frac{d}{d t} \int_{\Omega}\left|\operatorname{curl} b_{t}\right|^{2} d x+\int_{\Omega}\left|\Delta b_{t}\right|^{2} d x \\
& \quad=\int_{\Omega}\left(u_{t} \cdot \nabla b+u \cdot \nabla b_{t}-b_{t} \cdot \nabla u-b \cdot \nabla u_{t}\right) \Delta b_{t} d x \\
& \quad \leq\left(\left\|u_{t}\right\|_{L^{6}}\|\nabla b\|_{L^{3}}+\|u\|_{L^{\infty}}\left\|\nabla b_{t}\right\|_{L^{2}}+\|\nabla u\|_{L^{3}}\left\|b_{t}\right\|_{L^{6}}+\|b\|_{L^{\infty}}\left\|\nabla u_{t}\right\|_{L^{2}}\right)\left\|\Delta b_{t}\right\|_{L^{2}} \\
& \quad \leq \frac{1}{4}\left\|\Delta b_{t}\right\|_{L^{2}}^{2}+C\left(\|u\|_{L^{\infty}}^{2}+\|\nabla u\|_{L^{3}}^{2}\right)\left\|\nabla b_{t}\right\|_{L^{2}}^{2}+C\left(\|b\|_{L^{\infty}}^{2}+\|\nabla b\|_{L^{3}}^{2}\right)\left\|\nabla u_{t}\right\|_{L^{2}}^{2} .
\end{aligned}
$$

Combining (4.15) and (4.16) and using (4.14) and (4.13), we have

$$
\int_{\Omega}\left(\left|\operatorname{curl} u_{t}\right|^{2}+\left|\operatorname{curl} b_{t}\right|^{2}\right) d x+\int_{t_{0}}^{t} \int_{\Omega}\left(\left|\Delta u_{t}\right|^{2}+\left|\Delta b_{t}\right|^{2}\right) d x d \tau \leq C(e+y)^{C_{0} \epsilon}
$$

On the other hand, it follows from (4.5), (4.6), (4.3), (4.17), and (4.14) that

$$
\begin{aligned}
\|u(t)\|_{H^{3}}+\|b(t)\|_{H^{3}} \leq & C\left(1+\|\Delta \omega\|_{L^{2}}+\|\Delta j\|_{L^{2}}\right) \\
\leq & C\left(1+\left\|\partial_{t} \omega+u \cdot \nabla \omega-\omega \cdot \nabla u-b \cdot \nabla j-\sum_{i} \nabla b_{i} \times \partial_{i} b\right\|_{L^{2}}\right. \\
& \left.+\left\|\partial_{t} j+u \cdot \nabla j-b \cdot \nabla \omega+\sum_{i} \nabla u_{i} \times \partial_{i} b-\sum_{i} \nabla b_{i} \times \partial_{i} u\right\|_{L^{2}}\right) \\
\leq & C(e+y(t))^{C_{0} \epsilon}
\end{aligned}
$$

which yields

$$
\|u\|_{L^{\infty}\left(0, T ; H^{3}\right)}+\|b\|_{L^{\infty}\left(0, T ; H^{3}\right)} \leq C,
$$

This completes the proof of Theorem 1.2. 


\section{Proof of Theorem 1.3}

We only need to establish a priori estimates.

First, it follows from (1.12) and (1.13) that

$$
\|\rho\|_{L^{\infty}\left(0, T ; L^{\infty}\right)} \leq C
$$

Testing (1.14) by $u$ and using (1.12) and (1.13), we see that

$$
\frac{1}{2} \frac{d}{d t} \int_{\Omega} \rho u^{2} d x+\int_{\Omega}|\nabla u|^{2} d x=\int_{\Omega}(b \cdot \nabla) b \cdot u d x
$$

and testing (1.15) by $b$ and using (1.12) and (1.16), we find that

$$
\frac{1}{2} \frac{d}{d t} \int_{\Omega}|b|^{2} d x+\int_{\Omega}|\operatorname{curl} b|^{2} d x=\int_{\Omega}(b \cdot \nabla) u \cdot b d x .
$$

Summing up (5.2) and (5.3), we get the well-known energy inequality

$$
\frac{1}{2} \frac{d}{d t} \int_{\Omega}\left(\rho|u|^{2}+|b|^{2}\right) d x+\int_{\Omega}\left(|\nabla u|^{2}+|\operatorname{curl} b|^{2}\right) d x \leq 0 .
$$

(I) Let (1.21) hold.

Testing (1.15) by $|b|^{p-2} b(2 \leq p<\infty)$, using (1.12), (2.2), (2.3), and (2.5), setting $\phi=|b|^{\frac{p}{2}}$, and using the Gagliardo-Nirenberg inequality [3]

$$
\|\phi\|_{L^{\frac{2 s}{s-2}, 2}} \leq C\|\phi\|_{L^{2}}^{1-\frac{3}{s}}\|\phi\|_{H^{1}}^{\frac{3}{s}} \quad \text { with } 3<s \leq \infty
$$

and the generalized Hölder inequality [7]

$$
\|f g\|_{L^{p, q}} \leq C\|f\|_{L^{p_{1}, q_{1}}}\|g\|_{L^{p_{2}, q_{2}}}
$$

with $\frac{1}{p}=\frac{1}{p_{1}}+\frac{1}{p_{2}}$ and $\frac{1}{q}=\frac{1}{q_{1}}+\frac{1}{q_{2}}$, we derive

$$
\begin{aligned}
\frac{1}{p} & \frac{d}{d t} \int_{\Omega}|b|^{p} d x+\frac{1}{2} \int_{\Omega}|b|^{p-2}|\nabla b|^{2} d x+\left.\left.4 \frac{p-2}{p^{2}} \int_{\Omega}|\nabla| b\right|^{\frac{p}{2}}\right|^{2} d x \\
& =-\int_{\partial \Omega}|b|^{p-2}(b \cdot \nabla) v \cdot b d \sigma+\int_{\Omega}(b \cdot \nabla) u \cdot|b|^{p-2} b d x \\
& \leq\|\nabla v\|_{L^{\infty}} \int_{\partial \Omega}|b|^{p} d \sigma-\sum_{i} \int_{\Omega} b_{i} u \partial_{i}\left(|b|^{p-2} b\right) d x \\
& \leq C \int_{\partial \Omega} \phi^{2} d \sigma+C \int_{\Omega}|u \phi \nabla \phi| d x \\
& \leq C \int_{\partial \Omega} \phi^{2} d \sigma+C\|u\|_{L_{W}^{s}}\|\phi\|_{L^{\frac{2 s}{s-2}}, 2}\|\nabla \phi\|_{L^{2}} \\
& \leq C\|\phi\|_{L^{2}}\|\phi\|_{H^{1}}+C\|u\|_{L_{w}^{s}}\|\phi\|_{L^{2}}^{1-\frac{3}{s}}\|\nabla \phi\|_{L^{2}}^{1+\frac{3}{s}} \\
& \leq 2 \frac{p-2}{p^{2}} \int_{\Omega}|\nabla \phi|^{2} d x+C\|\phi\|_{L^{2}}^{2}+C\|u\|_{L_{w}^{s}}^{\frac{2 s}{s-3}}\|\phi\|_{L^{2}}^{2},
\end{aligned}
$$


which yields

$$
\begin{aligned}
\frac{d}{d t} \int_{\Omega} \phi^{2} d x+C \int_{\Omega}|\nabla \phi|^{2} d x & \leq C\left(1+\|u\|_{L_{w}^{s}}^{\frac{2 s}{s-3}}\right)\|\phi\|_{L^{2}}^{2} \\
& \leq C\left(1+\frac{\|u\|_{L_{W}^{s}}^{\frac{2 s}{s-3}}}{1+\log \left(e+\|u\|_{L_{W}^{s}}\right)}\right)\|\phi\|_{L^{2}}^{2}\left(1+\log \left(e+\|u\|_{L_{w}^{s}}\right)\right) \\
& \leq C\left(1+\frac{\|u\|_{L_{W}^{s}}^{\frac{2 s}{s-3}}}{1+\log \left(e+\|u\|_{L_{W}^{s}}^{s}\right)}\right)(1+\log (e+y))\|\phi\|_{L^{2}}^{2},
\end{aligned}
$$

from which it follows that

$$
\|b\|_{L^{\infty}\left(t_{0}, t ; L^{p}\right)}+\int_{t_{0}}^{t} \int_{\Omega}|b|^{2}|\nabla b|^{2} d x d \tau \leq C(e+y(t))^{C_{0} \epsilon}
$$

with

$$
y(t):=\sup _{\left[t_{0}, t\right]}\|u\|_{W^{1,4}}
$$

for any $0<t_{0} \leq t \leq T$, where $C_{0}$ is an absolute constant, provided that

$$
\int_{t_{0}}^{T} \frac{\|u\|_{L_{W}^{s}}^{\frac{2 s}{s-3}}}{1+\log \left(e+\|u\|_{L_{W}^{s}}\right)} d \tau \leq \epsilon \ll 1 .
$$

Testing (1.14) by $u_{t}$ and using (1.12) and (1.13), we infer that

$$
\begin{aligned}
\frac{1}{2} \frac{d}{d t} \int_{\Omega}|\nabla u|^{2} d x+\int_{\Omega} \rho\left|u_{t}\right|^{2} d x & =-\int_{\Omega} \rho u \cdot \nabla u \cdot u_{t} d x+\int_{\Omega} b \cdot \nabla b \cdot u_{t} d x \\
& =: I_{1}+I_{2} .
\end{aligned}
$$

We first compute $I_{2}$ :

$$
\begin{aligned}
I_{2} & =\int_{\Omega} \operatorname{div}(b \otimes b) \cdot u_{t} d x=-\int_{\Omega} b \otimes b: \nabla u_{t} d x \\
& =-\frac{d}{d t} \int_{\Omega} b \otimes b: \nabla u d x+2 \int_{\Omega} b \otimes b_{t}: \nabla u d x \\
& \leq-\frac{d}{d t} \int_{\Omega} b \otimes b: \nabla u d x+C\left\|b_{t}\right\|_{L^{2}}\|b\|_{L^{6}}\|\nabla u\|_{L^{3}} \\
& \leq-\frac{d}{d t} \int_{\Omega} b \otimes b: \nabla u d x+C\left\|b_{t}\right\|_{L^{2}}\|b\|_{L^{6}}\|\nabla u\|_{L^{2}}^{\frac{1}{2}}\|u\|_{H^{2}}^{\frac{1}{2}} \\
& \leq-\frac{d}{d t} \int_{\Omega} b \otimes b: \nabla u d x+\delta\left\|b_{t}\right\|_{L^{2}}^{2}+\delta\|u\|_{H^{2}}^{2}+C\|b\|_{L^{6}}^{4}\|\nabla u\|_{L^{2}}^{2}
\end{aligned}
$$

for any $0<\delta<1$.

We use (5.1), (5.5), and (5.6) to bound $I_{1}$ as follows:

$$
I_{1} \leq\left\|\sqrt{\rho} u_{t}\right\|_{L^{2}}\|\sqrt{\rho}\|_{L^{\infty}}\|u\|_{L_{W}^{s}}\|\nabla u\|_{L^{\frac{2 s}{s-2}, 2}}
$$




$$
\begin{aligned}
& \leq C\left\|\sqrt{\rho} u_{t}\right\|_{L^{2}}\|u\|_{L_{w}^{s}}\|\nabla u\|_{L^{2}}^{1-\frac{3}{s}}\|u\|_{H^{2}}^{\frac{3}{s}} \\
& \leq \delta\left\|\sqrt{u_{t}}\right\|_{L^{2}}^{2}+\delta\|u\|_{H^{2}}^{2}+C\|u\|_{L_{W}^{s}}^{\frac{2 s}{s-3}}\|\nabla u\|_{L^{2}}^{2}
\end{aligned}
$$

for any $0<\delta<1$.

On the other hand, by the $H^{2}$-theory of the Stokes system, using (5.1), (5.5), and (5.6), we obtain

$$
\begin{aligned}
\|u\|_{H^{2}} & \leq C\left\|-\Delta u+\nabla\left(\pi+\frac{1}{2}|b|^{2}\right)\right\|_{L^{2}} \\
& \leq C\left\|\rho \partial_{t} u+\rho u \cdot \nabla u-b \cdot \nabla b\right\|_{L^{2}} \\
& \leq C\left\|\sqrt{\rho} u_{t}\right\|_{L^{2}}+C\|u\|_{L_{w}^{s}}^{s}\|\nabla u\|_{L^{\frac{2 s}{s-2}, 2}}+C\|b \cdot \nabla b\|_{L^{2}} \\
& \leq C\left\|\sqrt{\rho} u_{t}\right\|_{L^{2}}+C\|u\|_{L_{w}^{s}}\|\nabla u\|_{L^{2}}^{1-\frac{3}{s}}\|u\|_{H^{2}}^{\frac{3}{s}}+C\|b \cdot \nabla b\|_{L^{2}},
\end{aligned}
$$

which gives

$$
\|u\|_{H^{2}} \leq C\left\|\sqrt{\rho} u_{t}\right\|_{L^{2}}+C\|b \cdot \nabla b\|_{L^{2}}+C\|u\|_{L_{W}^{s}}^{\frac{s}{s-3}}\|\nabla u\|_{L^{2}} .
$$

Testing (1.15) by $b_{t}-\Delta b$ and using (5.5) and (5.6), we deduce that

$$
\begin{aligned}
& \frac{d}{d t} \int_{\Omega}|\operatorname{curl} b|^{2} d x+\int_{\Omega}\left(\left|b_{t}\right|^{2}+|\Delta b|^{2}\right) d x \\
& \quad=\int_{\Omega}(b \cdot \nabla u-u \cdot \nabla b)\left(b_{t}-\Delta b\right) d x \\
& \quad \leq\left(\|u\|_{L_{w}^{s}}\|\nabla b\|_{L^{\frac{2 s}{s-2}, 2}}+\|b\|_{L^{6}}\|\nabla u\|_{L^{3}}\right)\left(\left\|b_{t}\right\|_{L^{2}}+\|\Delta b\|_{L^{2}}\right) \\
& \quad \leq C\left(\|u\|_{L_{w}^{s}}\|\nabla b\|_{L^{2}}^{1-\frac{3}{s}}\|b\|_{H^{2}}^{\frac{3}{s}}+C\|b\|_{L^{6}}\|\nabla u\|_{L^{2}}^{\frac{1}{2}}\|u\|_{H^{2}}^{\frac{1}{2}}\right)\left(\left\|b_{t}\right\|_{L^{2}}+\|\Delta b\|_{L^{2}}\right) \\
& \quad \leq \frac{1}{2}\left(\left\|b_{t}\right\|_{L^{2}}^{2}+\|\Delta b\|_{L^{2}}^{2}\right)+\delta\|u\|_{H^{2}}^{2}+C\|b\|_{L^{6}}^{4}\|\nabla u\|_{L^{2}}^{2}+C\|u\|_{L_{W}^{s}}^{\frac{2 s}{s-3}}\|\nabla b\|_{L^{2}}^{2}+C
\end{aligned}
$$

for any $0<\delta<1$.

It is easy to compute that

$$
\begin{aligned}
\frac{d}{d t} \int_{\Omega}|b|^{4} d x & \leq C \int_{\Omega}|b|^{3}\left|b_{t}\right| d x \\
& \leq C\|b\|_{L^{6}}^{3}\left\|b_{t}\right\|_{L^{2}} \leq \delta\left\|b_{t}\right\|_{L^{2}}^{2}+C\|b\|_{L^{6}}^{6}
\end{aligned}
$$

for any $0<\delta<1$.

Combining (5.9), (5.10), (5.11), (5.12), (5.13) and (5.14), and taking $\delta$ small enough, we obtain

$$
\begin{aligned}
& \frac{d}{d t} \int_{\Omega}\left(|\nabla u|^{2}+|\operatorname{curl} b|^{2}+b \otimes b: \nabla u+C_{0}|b|^{4}\right) d x \\
& \quad+\int_{\Omega}\left(\rho\left|u_{t}\right|^{2}+\left|b_{t}\right|^{2}+|\Delta b|^{2}\right) d x+\|u\|_{H^{2}}^{2} \\
& \quad \leq C\|b\|_{L^{6}}^{4}\|\nabla u\|_{L^{2}}^{2}+C\|u\|_{L_{w}^{s}}^{\frac{2 s}{S-3}}\left(\|\nabla u\|_{L^{2}}^{2}+\|\operatorname{curl} b\|_{L^{2}}^{2}\right)+C\|b \cdot \nabla b\|_{L^{2}}^{2}+C .
\end{aligned}
$$


Using (5.4), (5.7), (5.8), and the Gronwall inequality, we have

$$
\begin{aligned}
& \int_{\Omega}\left(|\nabla u|^{2}+|\operatorname{curl} b|^{2}+b \otimes b: \nabla u+C_{0}|b|^{4}\right) d x \\
& \leq\left[\int_{\Omega}\left(\left|\nabla u_{0}\right|^{2}+\left|\operatorname{curl} b_{0}\right|^{2}+b_{0} \otimes b_{0}: \nabla u_{0}+C_{0}\left|b_{0}\right|^{4}\right) d x\right. \\
& \left.\quad+C\|b\|_{L^{\infty}\left(t_{0}, t ; L^{6}\right)}^{4} \int_{t_{0}}^{t}\|\nabla u\|_{L^{2}}^{2} d \tau+C\left(t-t_{0}\right)+C \int_{t_{0}}^{t}\|b \cdot \nabla b\|_{L^{2}}^{2} d \tau\right] \\
& \quad \times \exp \left(\int_{t_{0}}^{t}\|u\|_{L_{w}^{s-3}}^{\frac{2 s}{s-3}} d \tau\right) \\
& \leq C(e+y)^{C_{0} \epsilon} \exp \left[\int_{t_{0}}^{t} \frac{\|u\|_{L_{w}^{s}}^{\frac{2 s}{s-3}}}{1+\log \left(e+\|u\|_{L_{W}^{s}}\right)} d \tau(1+\log (e+y))\right] \\
& \leq C(e+y)^{C_{0} \epsilon} .
\end{aligned}
$$

Plugging (5.16) into (5.15) and integrating over $\left[t_{0}, t\right]$, we have

$$
\int_{t_{0}}^{t} \int_{\Omega}\left(\rho\left|u_{t}\right|^{2}+\left|b_{t}\right|^{2}+|\Delta b|^{2}\right) d x d \tau+\int_{t_{0}}^{t}\|u\|_{H^{2}}^{2} d \tau \leq C(e+y)^{C_{0} \epsilon} .
$$

Applying $\partial_{t}$ to (1.15), testing by $u_{t}$, and using (1.12) and (1.13), we obtain

$$
\begin{aligned}
& \frac{1}{2} \frac{d}{d t} \int_{\Omega} \rho\left|u_{t}\right|^{2} d x+\int_{\Omega}\left|\nabla u_{t}\right|^{2} d x \\
&=-\int_{\Omega} \rho u \cdot \nabla\left|u_{t}\right|^{2} d x-\int_{\Omega} \rho u \cdot \nabla\left(u \cdot \nabla u \cdot u_{t}\right) d x \\
& \quad-\int_{\Omega} \rho u_{t} \cdot \nabla u \cdot u_{t} d x+\int_{\Omega} b \otimes b_{t}: \nabla u_{t} d x+\int_{\Omega} b_{t} \otimes b: \nabla u_{t} d x \\
& \leq C\|u\|_{L^{6}}\left\|\sqrt{\rho} u_{t}\right\|_{L^{3}}\left\|\nabla u_{t}\right\|_{L^{2}}+C\|u\|_{L^{6}}\|\nabla u\|_{L^{6}}\left\|u_{t}\right\|_{L^{6}}\|\nabla u\|_{L^{2}} \\
&+C\|u\|_{L^{6}}^{2}\|\Delta u\|_{L^{2}}\left\|u_{t}\right\|_{L^{6}}+C\|u\|_{L^{6}}^{2}\|\nabla u\|_{L^{6}}\left\|\nabla u_{t}\right\|_{L^{2}} \\
&+C\left\|\sqrt{\rho} u_{t}\right\|_{L^{4}}^{2}\|\nabla u\|_{L^{2}}+C\|b\|_{L^{6}}\left\|b_{t}\right\|_{L^{3}}\left\|\nabla u_{t}\right\|_{L^{2}} \\
& \leq C\|\nabla u\|_{L^{2}}\left\|\sqrt{\rho} u_{t}\right\|_{L^{2}}^{\frac{1}{2}}\left\|\sqrt{\rho} u_{t}\right\|_{L^{6}}^{\frac{1}{2}}\left\|\nabla u_{t}\right\|_{L^{2}} \\
&+C\|\nabla u\|_{L^{2}}^{2}\|u\|_{H^{2}}\left\|\nabla u_{t}\right\|_{L^{2}}+C\|\nabla u\|_{L^{2}}\left\|\sqrt{\rho} u_{t}\right\|_{L^{2}}^{\frac{1}{2}}\left\|\sqrt{\rho} u_{t}\right\|_{L^{6}}^{\frac{3}{2}} \\
&+C\|b\|_{L^{6}}\left\|b_{t}\right\|_{L^{3}}\left\|\nabla u_{t}\right\|_{L^{2}} \\
& \leq C\|\nabla u\|_{L^{2}}\left\|\sqrt{\rho} u_{t}\right\|_{L^{2}}^{\frac{1}{2}}\left\|\nabla u_{t}\right\|_{L^{2}}^{\frac{3}{2}}+C\|\nabla u\|_{L^{2}}^{2}\|u\|_{H^{2}}\left\|\nabla u_{t}\right\|_{L^{2}} \\
&+C\|\nabla u\|_{L^{2}}\left\|\sqrt{\rho} u_{t}\right\|_{L^{2}}^{\frac{1}{2}}\left\|\nabla u_{t}\right\|_{L^{2}}^{\frac{3}{2}}+C\|b\|_{L^{6}}\left\|b_{t}\right\|_{L^{3}}\left\|\nabla u_{t}\right\|_{L^{2}} \\
& \leq \frac{1}{4}\left\|\nabla u_{t}\right\|_{L^{2}}^{2}+C\|\nabla u\|_{L^{2}}^{4}\left(\left\|\sqrt{\rho} u_{t}\right\|_{L^{2}}^{2}+\|u\|_{H^{2}}^{2}\right)+C\|b\|_{L^{6}}^{2}\left\|b_{t}\right\|_{L^{3}}^{2} \\
& \leq \frac{1}{4}\left\|\nabla u_{t}\right\|_{L^{2}}^{2}+C\|\nabla u\|_{L^{2}}^{4}\left(\left\|\sqrt{\rho} u_{t}\right\|_{L^{2}}^{2}+\|u\|_{H^{2}}^{2}\right)+\frac{1}{4}\left\|\operatorname{curl} b_{t}\right\|_{L^{2}}^{2} \\
&+C\|b\|_{L^{6}}^{4}\left\|b_{t}\right\|_{L^{2}}^{2} . \\
&
\end{aligned}
$$


Applying $\partial_{t}$ to (1.15), testing by $b_{t}$, and using (1.12), we get

$$
\begin{aligned}
& \frac{1}{2} \frac{d}{d t} \int_{\Omega}\left|b_{t}\right|^{2} d x+\int_{\Omega}\left|\operatorname{curl} b_{t}\right|^{2} d x \\
& \quad=-\int_{\Omega}\left(u_{t} \cdot \nabla b-b_{t} \nabla u-b \cdot \nabla u_{t}\right) b_{t} d x \\
& \quad \leq\left\|u_{t}\right\|_{L^{6}}\|\nabla b\|_{L^{2}}\left\|b_{t}\right\|_{L^{3}}+\|\nabla u\|_{L^{2}}\left\|b_{t}\right\|_{L^{4}}^{2}+\left\|\nabla u_{t}\right\|_{L^{2}}\|b\|_{L^{6}}\left\|b_{t}\right\|_{L^{3}} \\
& \quad \leq \frac{1}{4}\left\|\nabla u_{t}\right\|_{L^{2}}^{2}+\frac{1}{4}\left\|\operatorname{curl} b_{t}\right\|_{L^{2}}^{2}+C\|\nabla b\|_{L^{2}}^{4}\left\|b_{t}\right\|_{L^{2}}^{2}+C\|\nabla u\|_{L^{2}}^{4}\left\|b_{t}\right\|_{L^{2}}^{2} .
\end{aligned}
$$

Combining (5.18) and (5.19) and integrating over $\left[t_{0}, t\right]$, we have

$$
\int_{\Omega}\left(\left|\rho u_{t}\right|^{2}+\left|b_{t}\right|^{2}\right) d x+\int_{t_{0}}^{t} \int_{\Omega}\left(\left|\nabla u_{t}\right|^{2}+\left|\operatorname{curl} b_{t}\right|^{2}\right) d x d \tau \leq C(e+y)^{C_{0} \epsilon} .
$$

Similarly to (5.12), we deduce that

$$
\begin{aligned}
\|u\|_{H^{2}} & \leq C\left\|\sqrt{\rho} u_{t}\right\|_{L^{2}}+C\|u\|_{L^{6}}\|\nabla u\|_{L^{3}}+C\|b\|_{L^{6}}\|\nabla b\|_{L^{3}} \\
& \leq C\left\|\sqrt{\rho} u_{t}\right\|_{L^{2}}+C\|u\|_{L^{6}}\|\nabla u\|_{L^{2}}^{\frac{1}{2}}\|u\|_{H^{2}}^{\frac{1}{2}}+C\|b\|_{L^{6}}\|\nabla b\|_{L^{2}}^{\frac{1}{2}}\|b\|_{H^{2}}^{\frac{1}{2}}
\end{aligned}
$$

which leads to

$$
\|u\|_{H^{2}}^{2} \leq C\left\|\sqrt{\rho} u_{t}\right\|_{L^{2}}^{2}+C\|\nabla u\|_{L^{2}}^{6}+C\|\nabla b\|_{L^{2}}^{6}+\frac{1}{2}\|b\|_{H^{2}}^{2} .
$$

Similarly, we have

$$
\begin{aligned}
\|b\|_{H^{2}} & \leq C\left\|b_{t}+u \cdot \nabla b-b \cdot \nabla u\right\|_{L^{2}} \\
& \leq C\left\|b_{t}\right\|_{L^{2}}+C\|u\|_{L^{6}}\|\nabla b\|_{L^{3}}+C\|b\|_{L^{6}}\|\nabla u\|_{L^{3}} \\
& \leq C\left\|b_{t}\right\|_{L^{2}}+C\|u\|_{L^{6}}\|\nabla b\|_{L^{2}}^{\frac{1}{2}}\|b\|_{H^{2}}^{\frac{1}{2}}+C\|b\|_{L^{6}}\|\nabla u\|_{L^{2}}^{\frac{1}{2}}\|u\|_{H^{2}}^{\frac{1}{2}},
\end{aligned}
$$

which implies

$$
\|b\|_{H^{2}}^{2} \leq C\left\|b_{t}\right\|_{L^{2}}^{2}+C\|\nabla u\|_{L^{2}}^{6}+C\|\nabla b\|_{L^{2}}^{6}+\frac{1}{2}\|u\|_{H^{2}}^{2} .
$$

Combining (5.21) and (5.22) and using (5.20) and (5.16), we conclude that

$$
\|u\|_{H^{2}}^{2}+\|b\|_{H^{2}}^{2} \leq C(e+y)^{C_{0} \epsilon}
$$

and thus

$$
\|u\|_{L^{\infty}\left(0, T ; H^{2}\right)}+\|b\|_{L^{\infty}\left(0, T ; H^{2}\right)} \leq C
$$

Now it is standard to prove that

$$
\|u\|_{L^{2}\left(0, T ; H^{3}\right)}+\|b\|_{L^{2}\left(0, T ; H^{3}\right)} \leq C
$$




$$
\|\rho\|_{L^{\infty}\left(0, T ; H^{2}\right)} \leq C \text {. }
$$

(II) Let (1.22) hold.

Similarly to (5.7), we take $s=\infty$ and using (2.4), we still get (5.7), provided that

$$
\int_{t_{0}}^{T}\|u(t)\|_{\mathrm{BMO}}^{2} d t \leq \epsilon \ll 1
$$

We still have (5.9), (5.10), (5.11) with $s=\infty$, (5.12) with $s=\infty$, (5.13) with $s=\infty$, and (5.14), (5.15) with $s=\infty$, and then using (5.27) and (2.4), we arrive at (5.16) and (5.17). Then by the same calculations as those in (5.18)-(5.26), we conclude that (5.18)-(5.26) hold.

This completes the proof of Theorem 1.3.

\section{Proof of Theorem 1.4}

We only need to establish a priori estimates.

First, using the formula $a \times(b \times c)=(a \cdot c) b-(a \cdot b) c$ and the fact that $|d|=1$ implies $d \Delta d=-|\nabla d|^{2}$, we have the following equivalent equation:

$$
\frac{1}{2} d_{t}-\frac{1}{2} d \times d_{t}=\Delta d+d|\nabla d|^{2}
$$

Testing (6.1) by $d_{t}$ and using $(a \times b) \cdot b=0$ and $d \cdot d_{t}=0$, we get

$$
\frac{d}{d t} \int_{\Omega}|\nabla d|^{2} d x+\int_{\Omega}\left|d_{t}\right|^{2} d x \leq 0
$$

Testing (1.23) by $-\Delta d_{t}$ and using $|d|=1$, we find that

$$
\begin{aligned}
& \frac{1}{2} \frac{d}{d t} \int_{\Omega}|\Delta d|^{2} d x+\int_{\Omega}\left|\nabla d_{t}\right|^{2} d x \\
& \quad=-\int_{\Omega}\left(d|\nabla d|^{2}+d \times \Delta d\right) \cdot \Delta d_{t} d x \\
& \quad=\int_{\Omega} \nabla\left(d|\nabla d|^{2}+d \times \Delta d\right) \cdot \nabla d_{t} d x \\
& \quad \leq C\left(\|\nabla d\|_{L^{q}}\|\nabla d\|_{L^{\frac{4 q}{q-2}}}^{2}+\|\nabla d\|_{L^{q}}\|\Delta d\|_{L^{\frac{2 q}{q-2}}}+\|\nabla \Delta d\|_{L^{2}}\right)\left\|\nabla d_{t}\right\|_{L^{2}} \\
& \quad \leq C\left(\|\nabla d\|_{L^{q}}\|\Delta d\|_{L^{\frac{2 q}{q-2}}}+\|\nabla \Delta d\|_{L^{2}}\right)\left\|\nabla d_{t}\right\|_{L^{2}} \\
& \quad \leq C\left(\|\nabla d\|_{L^{q}}\|\Delta d\|_{L^{2}}^{1-\frac{3}{q}}\|d\|_{H^{3}}^{\frac{3}{q}}+\|d\|_{H^{3}}\right)\left\|\nabla d_{t}\right\|_{L^{2}} \\
& \quad \leq \frac{1}{4}\left\|\nabla d_{t}\right\|_{L^{2}}^{2}+\delta\|d\|_{H^{3}}^{2}+C\|\nabla d\|_{L^{q}}^{\frac{2 q}{q-3}}\|\Delta d\|_{L^{2}}^{2}
\end{aligned}
$$

for any $0<\delta<1$. Here we have used the Gagliardo-Nirenberg inequalities

$$
\begin{aligned}
& \|\nabla d\|_{L^{\frac{4 q}{q-2}}}^{2} \leq C\|d\|_{L^{\infty}}\|\Delta d\|_{L^{\frac{2 q}{q-2}}}, \\
& \|\Delta d\|_{L^{\frac{2 q}{q-2}}} \leq C\|\Delta d\|_{L^{2}}^{1-\frac{3}{q}}\|d\|_{H^{3}}^{\frac{3}{q}} .
\end{aligned}
$$


Applying $\partial_{i}$ to (1.23), we get

$$
\partial_{i} d_{t}-\Delta \partial_{i} d=\partial_{i}\left(d|\nabla d|^{2}\right)+\partial_{i} d \times \Delta d+d \times \Delta \partial_{i} d
$$

Testing this equation by $\Delta \partial_{i} d$, summing over $i$, and using (6.4) and (6.5) and $|d|=1$, we obtain

$$
\begin{aligned}
\|d\|_{H^{3}} & \leq C\left(\|d\|_{L^{2}}+\|\nabla \Delta d\|_{L^{2}}\right) \\
& \leq C+C\left\|\nabla d_{t}\right\|_{L^{2}}+C\left\|\nabla\left(d|\nabla d|^{2}\right)\right\|_{L^{2}}+\sum_{i} C\left\|\partial_{i} d \times \Delta d\right\|_{L^{2}} \\
& \leq C+C\left\|\nabla d_{t}\right\|_{L^{2}}+C\|\nabla d\|_{L^{q}}\|\nabla d\|_{L^{\frac{4 q}{q-2}}}^{2}+C\|\nabla d\|_{L^{q}}\|\Delta d\|_{L^{\frac{2 q}{q-2}}} \\
& \leq C+C\left\|\nabla d_{t}\right\|_{L^{2}}+C\|\nabla d\|_{L^{q}}\|\Delta d\|_{L^{\frac{2 q}{q-2}}} \\
& \leq C+C\left\|\nabla d_{t}\right\|_{L^{2}}+C\|\nabla d\|_{L^{q}}\|\Delta d\|_{L^{2}}^{1-\frac{3}{q}}\|d\|_{H^{3}}^{\frac{3}{q}},
\end{aligned}
$$

which yields

$$
\|d\|_{H^{3}} \leq C+C\left\|\nabla d_{t}\right\|_{L^{2}}+C\|\nabla d\|_{L^{q}}^{\frac{q}{q-3}}\|\Delta d\|_{L^{2}}
$$

Plugging (6.6) into (6.3) and taking $\delta$ small enough, we have

$$
\begin{aligned}
& \frac{d}{d t} \int_{\Omega}|\Delta d|^{2} d x+\int_{\Omega}\left|\nabla d_{t}\right|^{2} d x \\
& \quad \leq C+C\|\nabla d\|_{L^{q}}^{\frac{2 q}{q-3}}\|\Delta d\|_{L^{2}}^{2} \\
& \quad \leq C+C \frac{\|\nabla d\|_{L^{q}}^{\frac{2 q}{q-3}}}{1+\log \left(e+\|\nabla d\|_{L^{q}}\right)}\|\Delta d\|_{L^{2}}^{2} \log \left(e+\|\nabla d\|_{L^{q}}\right) \\
& \quad \leq C+C \frac{\|\nabla d\|_{L^{q}}^{\frac{2 q}{q-3}}}{1+\log \left(e+\|\nabla d\|_{L^{q}}\right)}\|\Delta d\|_{L^{2}}^{2} \log (e+y),
\end{aligned}
$$

which implies

$$
\int_{\Omega}|\Delta d|^{2} d x+\int_{t_{0}}^{t} \int_{\Omega}\left|\nabla d_{t}\right|^{2} d x d \tau \leq C(e+y)^{C_{0} \epsilon}
$$

provided that

$$
\int_{t_{0}}^{T} \frac{\|\nabla d\|_{L^{q}}^{\frac{2 q}{q-3}}}{1+\log \left(e+\|\nabla d\|_{L^{q}}\right)} d \tau \leq \epsilon \ll 1
$$

with $y(t):=\sup _{\left[t_{0}, t\right]}\|d\|_{H^{3}}$ for any $0<t_{0} \leq t \leq T$, where $C_{0}$ is an absolute constant.

It follows from (1.23), (6.6), and (6.7) that

$$
\int_{\Omega}\left|d_{t}\right|^{2} d x+\int_{t_{0}}^{t}\|d\|_{H^{3}}^{2} d \tau \leq C(e+y)^{C_{0} \epsilon}
$$


Applying $\partial_{t}$ to (1.23), testing by $-\Delta d_{t}$, and using $|d|=1,(6.7)$, and (6.8), we have

$$
\begin{aligned}
\frac{1}{2} & \frac{d}{d t} \int_{\Omega}\left|\nabla d_{t}\right|^{2} d x+\int_{\Omega}\left|\Delta d_{t}\right|^{2} d x \\
& =-\int_{\Omega}\left[\partial_{t}\left(d|\nabla d|^{2}\right)+d_{t} \times \Delta d\right] \Delta d_{t} d x \\
& \leq C\left(\|\nabla d\|_{L^{6}}^{2}\left\|d_{t}\right\|_{L^{6}}+\|\nabla d\|_{L^{6}}\left\|\nabla d_{t}\right\|_{L^{3}}+\left\|d_{t}\right\|_{L^{\infty}}\|\Delta d\|_{L^{2}}\right)\left\|\Delta d_{t}\right\|_{L^{2}} \\
& \leq C\left(\|\nabla d\|_{L^{6}}^{2}\left\|d_{t}\right\|_{L^{6}}+\|\Delta d\|_{L^{2}}\left\|\nabla d_{t}\right\|_{L^{2}}^{\frac{1}{2}}\left\|\Delta d_{t}\right\|_{L^{2}}^{\frac{1}{2}}+\|\Delta d\|_{L^{2}}\left\|d_{t}\right\|_{L^{2}}\right)\left\|\Delta d_{t}\right\|_{L^{2}} \\
& \leq \frac{1}{2}\left\|\Delta d_{t}\right\|_{L^{2}}^{2}+C\|d\|_{H^{2}}^{4}\left\|d_{t}\right\|_{H^{1}}^{2}+C\|d\|_{H^{2}}^{2}\left\|d_{t}\right\|_{L^{2}}^{2},
\end{aligned}
$$

which implies

$$
\int_{\Omega}\left|\nabla d_{t}\right|^{2} d x+\int_{t_{0}}^{t}\left\|\Delta d_{t}\right\|_{L^{2}}^{2} d \tau \leq C(e+y)^{C_{0} \epsilon}
$$

It follows from (6.6), (6.7), (6.8), and (6.9) that

$$
\|d\|_{H^{3}} \leq C+C\left\|\nabla d_{t}\right\|_{L^{2}}+C\|\nabla d\|_{L^{6}}^{2}\|\Delta d\|_{L^{2}} \leq C(e+y)^{C_{0} \epsilon},
$$

which leads to

$$
\|d\|_{L^{\infty}\left(0, T ; H^{3}\right)} \leq C .
$$

This completes the proof of Theorem 1.4.

\section{Competing interests}

The authors declare that they have no competing interests.

\section{Authors' contributions}

The authors declare that the study was realized in collaboration with the same responsibility. All authors read and approved the final manuscript.

\section{Author details}

${ }^{1}$ Department of Applied Mathematics, Nanjing Forestry University, Nanjing, 210037, P.R. China. ${ }^{2}$ Institute of Applied Physics and Computational Mathematics, Beijing, 100088, P.R. China.

\section{Acknowledgements}

J. Fan is partially supported by NSFC (No. 11171154), Junpin Yin is supported by the NSFC (Grant No. U1430103) and Beijing Center for Mathematics and Information Interdisciplinary Sciences (BCMIIS). The authors would like to thank the referee for reading the paper carefully and for the valuable comments, which improved the presentation of the paper.

Received: 14 January 2016 Accepted: 21 April 2016 Published online: 29 April 2016

\section{References}

1. Giga, Y: Solutions for semilinear parabolic equations in $L^{p}$ and regularity of weak solutions of the Navier-Stokes system. J. Differ. Equ. 62, 186-212 (1986)

2. Kim, H: A blow-up criterion for the nonhomogeneous incompressible Navier-Stokes equations. SIAM J. Math. Anal. 37, 1417-1434 (2006)

3. Kang, K, Kim, J: Regularity criteria of the magnetohydrodynamic equations in bounded domains or a half space. J. Differ. Equ. 253(2), 764-794 (2012)

4. Sermange, $M$, Temam, R: Some mathematical questions related to the MHD equations. Commun. Pure Appl. Math. 36(5), 635-664 (1983)

5. Chen, Q, Miao, C, Zhang, Z: The Beale-Kato-Majda criterion to the 3D magneto-hydrodynamics equations. Commun. Math. Phys. 275, 861-872 (2007)

6. Kozono, H, Ogawa, T, Taniuchi, Y: The critical Sobolev inequalities in Besov spaces and regularity criterion to some semilinear evolution equations. Math. Z. 242, 251-278 (2002) 
7. Triebel, H: Theory of Function Spaces. Birkhäuser, Basel (1983)

8. Wu, H: Strong solution to the incompressible MHD equations with vacuum. Comput. Math. Appl. 61, 2742-2753 (2011)

9. Carbou, G, Fabrie, P: Regular solutions for Landau-Lifshitz equation in a bounded domain. Differ. Integral Equ. 14, 213-229 (2001)

10. Fan, J, Ozawa, T: Logarithmically improved regularity criteria for Navier-Stokes and related equations. Math. Methods Appl. Sci. 32, 2309-2318 (2009)

11. Ogawa, T, Taniuchi, Y: A note on blow-up criterion to the 3D Euler equations in a bounded domain. J. Differ. Equ. 190 39-63 (2003)

12. Beirão da Veiga, $\mathrm{H}$, Cripo, F: Sharp inviscid limit results under Navier type boundary conditions. An $L^{p}$ theory. J. Math. Fluid Mech. 12, 397-411 (2010)

13. Adams, RA, Fournier, JF: Sobolev Spaces, 2nd edn. Pure and Appl. Math. (Amsterdam), vol. 140. Elsevier, Amsterdam (2003)

14. Lunardi, A: Interpolation Theory, 2nd edn. Lecture Notes. Scuola Normale Superiore di Pisa (New Series). Edizioni della Normale, Pisa (2009)

15. Ogawa, T: Sharp Sobolev inequality of logarithmic type and the limiting regularity condition to the harmonic heat flow. SIAM J. Math. Anal. 34, 1318-1330 (2003)

Submit your manuscript to a SpringerOpen ${ }^{\circ}$ journal and benefit from:

- Convenient online submission

- Rigorous peer review

- Immediate publication on acceptance

- Open access: articles freely available online

- High visibility within the field

- Retaining the copyright to your article

Submit your next manuscript at $\gg$ springeropen.com 